\title{
Feasibility study: non-invasive hemodynamic monitoring during cesarean section and intraoperative fluidic management.
}

Eleonora Pedrazzoli1 ${ }^{1}$ Katia Donadello ${ }^{1}$, Davide Cigolini ${ }^{1}$, Leonardo Gottin ${ }^{2}$ and Enrico Polati ${ }^{1}$

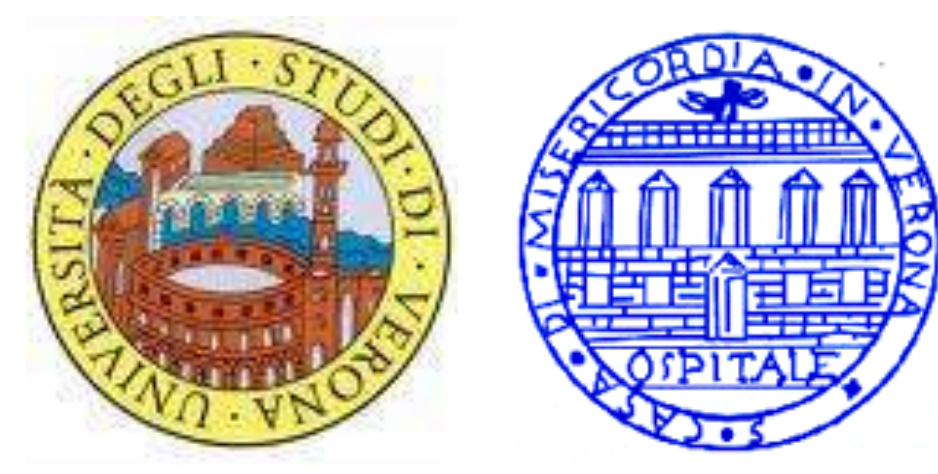

${ }^{1}$ Anesthesia and Intensive Care B Unit, Department of Surgery, Dentistry, Paediatrics and Gynaecology, University of Verona, AOUI-University Hospital Integrated Trust of Verona, 37121 - Verona (Italy)

${ }^{2}$ Cardio-Thoracic Anesthesia and Intensive Care Unit, Department of Surgery, Dentistry, Paediatrics and Gynaecology, University of Verona, AOUI-University Hospital Integrated Trust of Verona, 37121 - Verona (Italy)

INTRODUCTION : The maternal and neonatal outcome secondary to caesarean section surgery has improved thanks to the evolution of surgical and anesthetic techniques. In the literature we approach the hypotension from ALR in two ways : a volume load administated before starting anesthesia (PRELOAD) or infusion of fluids only during the execution of anesthesia (COLOAD). For both there is no standardization. AIMS : Observational study to test the use of advanced non-invasive hemodynamic monitoring comparing 2 types of volemic management of the patient undergoing caesarean section under subarachnoid anesthesia. METHODS : Pregnant patients (1840 years), single fetus, gestational age $>36 \mathrm{w}$, ASA I-II classes, under ordinary hospitalization were included. The patients were divided into 2 groups depending on the choice of the present anesthesiologist. PRELOAD: administration of $1 \mathrm{~L}$ of balanced solution pre-intervention, followed by other $500 \mathrm{cc}$ intraoperative ; COLOAD: $500 \mathrm{cc}$ iv of balanced solution infusion started during ALR execution. Vasopressor drugs ( $P A M<70 \mathrm{mmHg}$ ) were included for both groups. Monitoring: ECG, blood pressure with measurements every 5 minutes, pulse oximeter and Clear-sight probe (EV1000-Edwards Lifescience). Precise way points were identified where a Valsalva maneuver was performed for 5 seconds to obviate the bias of the measurements that are affected by changes in the respiratory cycle: entry into the operating room, after eventual volume filling, after ALR supine , incision, fetal and secondary extraction. RESULTS : We evaluated 18 patients, median age 35.5 (34.3-38.5) and 31 (30-33) years for the Coload groups $(n=10)$ and Preload $(n=8)$, respectively $(p<0.05)$. The two groups showed no significant differences in height, weight, ASA class, comorbidity, home therapy, gestational week and CT indication. The execution of the subarachnoid anesthesia was found to be the same in the 2 groups. Pre-operative $\mathrm{Hb}$ was 11.2 (10.7-11.5) vs $12.2(11.8-12.9) \mathrm{g} \mathrm{/} \mathrm{L}$ for the Coload and Preload groups, respectively $(p=0.06)$. Blood losses were not significantly different ( $p$ $=0.78$ ), as were the outcome of newborns (APGAR at I and V minute, umbilical blood gas values, weight in I and III day). The haemodynamic variables (Tab 3 and Fig 1-2-3-4)t were not significantly differentiated between the 2 groups under examination.
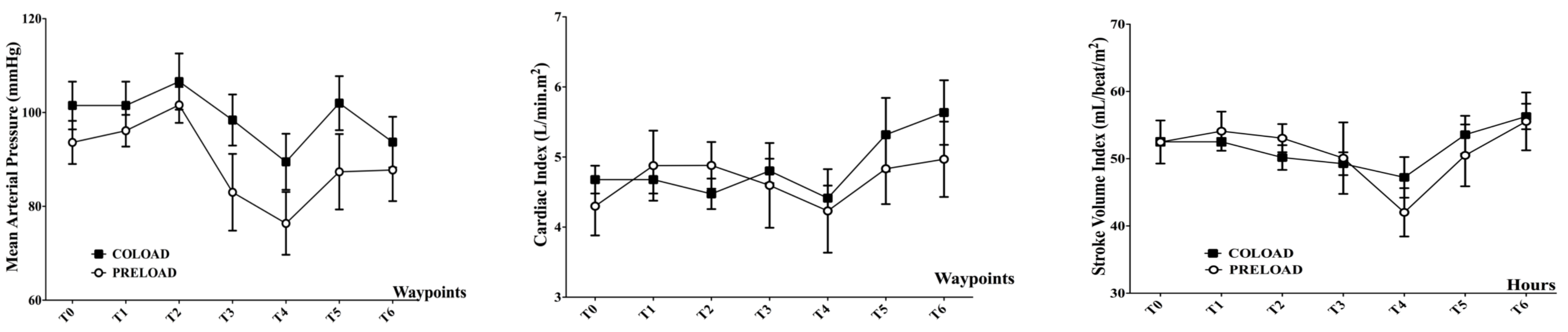

Not even the characteristics of the newborns were different.(Tab 4) CONCLUSIONS : The use of a completely non-invasive hemodynamic monitoring mode is proven feasible, reliable and well tolerated. Preliminary data from our study would indicate that the different fluid management does not significantly modify the hemodynamic parameters of the pregnant woman, nor the conditions of the newborn. This would confirm our hypothesis that the volemic filling performed before the operation would not be effective in terms of lasting improvement of cardiac output (rapid redistribution) and would not favorably influence the maternal and fetal state.

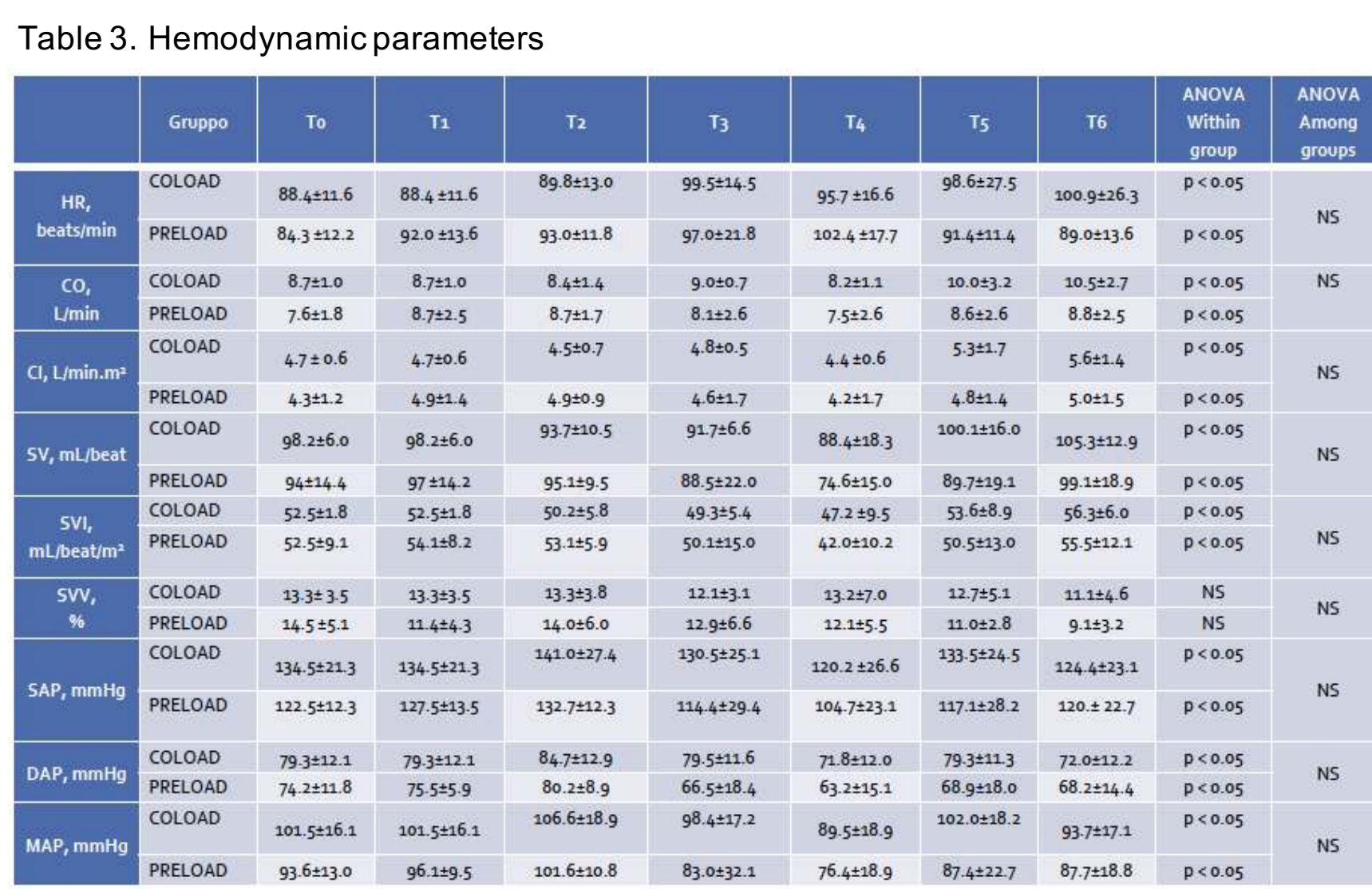

Table.4 Outcom of the newborn: Apgar index, weight and blood gas values of umbilical vessels

\begin{tabular}{|c|c|c|c|c|c|c|c|}
\hline & \multicolumn{3}{|c|}{ Coload group $(n=10)$} & \multicolumn{3}{|c|}{ Preload group $\{n=8\}$} & Pvalue \\
\hline APGAR 1:min & 9 & 9 & 9 & 9 & 8,75 & 9 & 0,42 \\
\hline APGAR $5^{\circ}$ min & 10 & 10 & 10 & 10 & 9,75 & 10 & 0,46 \\
\hline \multicolumn{8}{|l|}{ UMBBILICAL ARTERY } \\
\hline $\mathrm{PH}$ & 7.31 & 7.27 & 7,32 & 7.32 & 7,27 & 7,34 & 0,98 \\
\hline $\mathrm{PCO} 2$ (mming) & 49 & 47,25 & 57,5 & 53,5 & 51 & 59,2 & 0.29 \\
\hline $\mathrm{PO2}$ (mmhg) & 10,5 & 7,5 & 15,75 & 11 & 8,75 & 12,5 & 0,66 \\
\hline $\mathrm{HCO} 3(\mathrm{mEq} / \mathrm{L})$ & 25,9 & 25,4 & 26,8 & 27,3 & 26,1 & 27,6 & 0,15 \\
\hline BE $\quad(\mathrm{mE} \alpha / L)$ & $-1,25$ & $-2,37$ & $-0,77$ & $-0,75$ & $-1,07$ & $-0,1$ & 0,41 \\
\hline \multicolumn{8}{|l|}{ UMBILICAL VEIN } \\
\hline $\mathrm{PH}$ & 7,36 & 7,33 & 7,37 & 7,36 & 7,33 & 7,39 & 0,92 \\
\hline PCO2 (mmhg) & 42 & 37,75 & 45,5 & 42,5 & 39,7 & 47,2 & 0,49 \\
\hline $\mathrm{PO} 2$ (mming) & 23 & 20,5 & 28,5 & 22,5 & 18,7 & 32 & 0,93 \\
\hline $\mathrm{HCO} 3(\mathrm{mEq} / \mathrm{L})$ & 23 & 22,2 & 24 & 24,3 & 23,7 & 24,8 & 0,18 \\
\hline BE $\quad(m E q / L)$ & $-2,35$ & $-3,25$ & -2 & $-1,95$ & $-2,52$ & $-0,8$ & 0,39 \\
\hline Weight th gg grams: & 3015 & 2812 & 3500 & 2999 & 2875 & 3165 & 0,51 \\
\hline Weight 3 \%gg grams & 2820 & 2575 & 3300 & 2875 & 2772 & 2960 & 0,53 \\
\hline
\end{tabular}

